\title{
Soliton solutions of an integrable nonlinear Schrödinger equation with quintic terms
}

\author{
A. Chowdury, D. J. Kedziora, A. Ankiewicz, and N. Akhmediev \\ Optical Sciences Group, Research School of Physics and Engineering, The Australian National University, Canberra ACT 0200, Australia
}

(Received 15 July 2014; published 26 September 2014)

\begin{abstract}
We present the fifth-order equation of the nonlinear Schrödinger hierarchy. This integrable partial differential equation contains fifth-order dispersion and nonlinear terms related to it. We present the Lax pair and use Darboux transformations to derive exact expressions for the most representative soliton solutions. This set includes two-soliton collisions and the degenerate case of the two-soliton solution, as well as beating structures composed of two or three solitons. Ultimately, the new quintic operator and the terms it adds to the standard nonlinear Schrödinger equation (NLSE) are found to primarily affect the velocity of solutions, with complicated flow-on effects. Furthermore, we present a new structure, composed of coincident equal-amplitude solitons, which cannot exist for the standard NLSE.
\end{abstract}

DOI: 10.1103/PhysRevE.90.032922

PACS number(s): 05.45.Yv, 42.65.Tg, 42.81.Qb

\section{INTRODUCTION}

During the past few decades, soliton-based research has expanded far beyond optics into a diverse array of fields, including oceanography [1], plasma physics [2], molecular biology [3], meteorology [4], geology [5], and nonlinear field theory [6]. The interdisciplinary spread of this concept is remarkable in its speed, considering that the first theoretical prediction of an optical soliton, subject to several perturbations occurring in a glass fiber, was reported by Hasegawa and Tappert only in 1973 [7]. Experimental verification followed several years later [8], and it was shown that pulse broadening could indeed be counterbalanced by the nonlinearity related to the index of refraction. The major result, from the perspective of theory, was that these ensuing pulse profiles could be well described by basic sech-type functions.

Nonetheless, the caveat remains that the simplest expression for a soliton is a consequence of the nonlinear Schrödinger equation (NLSE), which is still only an approximation to the physics that governs wave evolution in various systems. Thus, to balance the analytic utility of these sech-type functions with heightened physical realism, better approximations have since been attained via the inclusion of additional phenomena, such as self-steepening, self-frequency shift, and third-order dispersion [9-12]. These are the next-lowest-order terms that have to be taken into account in studies of pulse propagation in optical fibers. These third-order terms are essential in supercontinuum generation [13], Cherenkov radiation by solitons [14], and pulse-deforming phenomena [15]. The role of fourth-order terms in a fiber also have been studied [16-18]. With ever-increasing intensity of the optical field and further shortening of pulses up to attosecond durations [19], the role of quintic terms is becoming ever more important. Generally speaking, the role of each of the terms of quintic order should be studied separately. However, there are many more quintic then cubic terms, and these studies cannot be carried out in a single step. Even clarifying the roles of the third-order terms required decades of research effort. The studies of quintic terms will need more effort, because of the higher number of them and the complexity of their contributions. Thus, it is a better idea first to find integrable models that can be studied analytically, because any other choice would require massive numerical modeling. Importantly, integrable models provide us with general forms of quintic terms, although restricting us in the choice of their relative contributions. Indeed, the new terms cannot be bundled onto the NLSE in any haphazard fashion but must be incorporated with certain parametric restrictions to maintain the integrability. On the other hand, the quintic terms remain independent of cubic and quartic terms.

In this work, we consider the part of the NLSE hierarchy that is most important for applications; it is given by the following:

$$
\begin{aligned}
& i \psi_{x}+S[\psi(x, t)]-i \alpha H[\psi(x, t)]+\gamma P[\psi(x, t)] \\
& \quad-i \delta Q[\psi(x, t)]=0,
\end{aligned}
$$

where the second-order $S$ is the nonlinear Schrödinger operator (including second-order dispersion),

$$
S[\psi(x, t)]=\frac{1}{2} \psi_{t t}+\psi|\psi|^{2},
$$

the third-order $H$ is the Hirota operator (beginning with thirdorder dispersion),

$$
H[\psi(x, t)]=\psi_{t t t}+6|\psi|^{2} \psi_{t},
$$

the fourth-order $P$ is the Lakshmanan-Porsezian-Daniel (LPD) operator (beginning with fourth-order dispersion),

$$
\begin{aligned}
P[\psi(x, t)]= & \psi_{t t t t}+8|\psi|^{2} \psi_{t t}+6 \psi|\psi|^{4}+4 \psi\left|\psi_{t}\right|^{2} \\
& +6 \psi_{t}^{2} \psi^{*}+2 \psi^{2} \psi_{t t}^{*},
\end{aligned}
$$

and the fifth-order $Q$ is the quintic operator (beginning with fifth-order dispersion),

$$
\begin{aligned}
Q[\psi(x, t)]= & \psi_{t t t t t}+10|\psi|^{2} \psi_{t t t}+30|\psi|^{4} \psi_{t}+10 \psi \psi_{t} \psi_{t t}^{*} \\
& +10 \psi \psi_{t}^{*} \psi_{t t}+20 \psi^{*} \psi_{t} \psi_{t t}+10 \psi_{t}^{2} \psi_{t}^{*} .
\end{aligned}
$$

More succinctly, we can write

$$
\begin{aligned}
Q[\psi(x, t)]= & \psi_{t t t t t}+10|\psi|^{2} \psi_{t t t}+10\left(\psi\left|\psi_{t}\right|^{2}\right)_{t} \\
& +20 \psi^{*} \psi_{t} \psi_{t t}+30|\psi|^{4} \psi_{t} .
\end{aligned}
$$

Here $x$ is the propagation variable and $t$ is the transverse variable ("time" in a moving frame), with the modulus of complex-valued $\psi(x, t)$ representing the envelope of the waves.

Within Eq. (1), the integrability of the fundamental NLS operator is best known. Various solitonic structures and families of other solution types have previously been presented for this 
system [20,21]. The simplest exactly solvable extension then arises when $\gamma=\delta=0$, known as the Hirota equation [22,23]. Accordingly, Painlevé analysis has been done for deformations of both the NLS and Hirota expressions [24]. Furthermore, when only $\alpha=\delta=0$, the system is also integrable and is known as the Lakshmanan-Porsezian-Daniel equation [25-27]. In this latter case, the coefficients of the additional terms were found using Painlevé analysis on a model of a Heisenberg spin chain but were independently derived elsewhere as part of the NLS hierarchy [28]. A different approach to these operators has since been developed, allowing for the construction of both solitons [29] and rogue waves [18] for arbitary $\alpha$ and $\gamma$ values.

The next sequential case of $\alpha=\gamma=0$ is our focus in this work, where Eq. (1) reduces to what will be called the "quintic NLSE." It has been written out previously [30], with emphasis that it is a member of the NLS integrable hierarchy [28]. An adjusted version of this equation with real $\psi$ has also already been presented, with Lax pair, conservation laws, and $N$-soliton solutions all generated as well [31]. However, along with its sixth-order relative for which symmetries have been investigated [32], it is regarded in this case as an extended Korteweg-de Vries equation, applicable to shallow-depth fluid studies. We shall instead provide the Lax pair for the full complex-valued quintic NLSE in Sec. II before utilizing the Darboux transformation scheme to derive exact expressions in Sec. III for both single- and double-soliton solutions. A brief investigation into triple-soliton solutions is also included in this work.

In fact, the hierarchy of integrable NLS equations is actually well-defined [28], where each sequential "order" attaches a collection of fixed-ratio terms to all those of the previous orders.

\section{LAX PAIRS}

The inverse scattering technique for the ordinary NLSE was developed in 1972 by Zakharov and Shabat [33]. This technique allows us to write the NLSE in terms of two matrix operators. Later studies have shown that a number of other integrable equations can be represented in similar form. The creation of the world of integrable evolution equations can be considered as one of the main achievements in mathematical physics of the 20th century [34].

Remarkably, Eq. (1) with quintic terms can also be structured in Lax pair format, meaning that it is integrable. Just as in the case of the NLSE, this form can be written as

$$
\frac{\partial R}{\partial t}=U R, \quad \frac{\partial R}{\partial x}=V R,
$$

such that the "zero-curvature" condition,

$$
U_{x}-V_{t}+[U, V]=0,
$$

returns the original system. Naturally, while this transformation has the downside of introducing new variables via the vector $R$, the upside is that the nonlinear system is now expressed as linear differential equations that are more readily solved by standard methods.

Importantly, Lax pairs also serve as the basis for many solution-generating procedures, ranging from the inverse scattering method to the Darboux scheme. In this work, we employ the latter.

Given this, we can consider two equations to be in the same Lax pair hierarchy if they share the same $U$ matrix. For instance, the standard NLSE is linked to the $2 \times 2$ AblowitzKaup-Newell-Segur (AKNS) matrix [35], given by

$$
U=i\left[\begin{array}{cc}
\lambda & \psi(x, t)^{*} \\
\psi(x, t) & -\lambda
\end{array}\right] .
$$

The general form of our quintic NLSE, which is thus named because the $V$ matrix is a fifth-order polynomial in eigenvalue $\lambda$, also uses Eq. (4) as its $U$ matrix. Specifically, the appropriate corresponding $V$ matrix is written as

$$
\begin{aligned}
& V=\sum_{j=0}^{5} \lambda^{j} V_{j}, \\
& V_{j}=i\left[\begin{array}{cc}
A_{j} & B_{j}^{*} \\
B_{j} & -A_{j}
\end{array}\right], \\
& A_{5}=16 \delta \text {, } \\
& B_{5}=0 \text {, } \\
& A_{4}=-8 \gamma \text {, } \\
& B_{4}=16 \delta \psi \text {, } \\
& A_{3}=-4 \alpha-8 \delta|\psi|^{2} \text {, } \\
& B_{3}=-8 \gamma \psi+8 i \delta \psi_{t}, \\
& A_{2}=1+4 \gamma|\psi|^{2}+4 i \delta\left(\psi_{t}^{*} \psi-\psi_{t} \psi^{*}\right) \text {, } \\
& B_{2}=-4 \alpha \psi-8 \delta|\psi|^{2} \psi-4 i \gamma \psi_{t}-4 \delta \psi_{t t} \text {, } \\
& A_{1}=f_{1}+2 \alpha|\psi|^{2}+6 \delta|\psi|^{4}-2 i \gamma\left(\psi_{t}^{*} \psi-\psi_{t} \psi^{*}\right) \\
& +2 \delta\left(\psi_{t t}^{*} \psi-\left|\psi_{t}\right|^{2}+\psi_{t t} \psi^{*}\right), \\
& B_{1}=\psi+4 \gamma|\psi|^{2} \psi-2 i \alpha \psi_{t}-12 i \delta|\psi|^{2} \psi_{t} \\
& +2 \gamma \psi_{t t}-2 i \delta \psi_{t t t}, \\
& A_{0}=\frac{1}{2} f_{0}-\frac{1}{2}|\psi|^{2}-3 \gamma|\psi|^{4}-i \alpha\left(\psi_{t}^{*} \psi-\psi_{t} \psi^{*}\right) \\
& -\gamma\left(\psi_{t t}^{*} \psi-\left|\psi_{t}\right|^{2}+\psi_{t t} \psi^{*}\right) \\
& -i \delta\left(\psi_{t t t}^{*} \psi-\psi_{t t}^{*} \psi_{t}+\psi_{t t} \psi_{t}^{*}-\psi_{t t t} \psi^{*}\right) \\
& -6 i \delta\left(\psi_{t}^{*} \psi-\psi_{t} \psi^{*}\right)|\psi|^{2} \\
& B_{0}=f_{1} \psi+2 \alpha|\psi|^{2} \psi+6 \delta|\psi|^{4} \psi+i \frac{1}{2} \psi_{t}+6 i \gamma|\psi|^{2} \psi_{t} \\
& +\alpha \psi_{t t}+2 \delta \psi_{t t}^{*} \psi^{2}+4 \delta\left|\psi_{t}\right|^{2} \psi+6 \delta\left(\psi_{t}\right)^{2} \psi^{*} \\
& +8 \delta \psi_{t t}|\psi|^{2}+i \gamma \psi_{t t t}+\delta \psi_{t t t t},
\end{aligned}
$$

where coefficients $f_{0}, f_{1}$ are arbitrary real constants while $\alpha$, $\gamma$, and $\delta$ are coefficients of the higher-order terms in Eq. (1). Thus, the $V$ matrix contains all necessary evolution data for the solutions of this equation. Naturally, when $\alpha=0, \gamma=0$, and $\delta=0$, the $V$ matrix describes the evolution data for the NLSE. 
With these $U$ and $V$ matrices, the zero-curvature equation reproduces

$$
\begin{aligned}
& i \psi_{x}-f_{0} \psi-i f_{1} \psi_{t}+\frac{1}{2}\left(\psi_{t t}+2|\psi|^{2} \psi\right)-i \alpha\left(\psi_{t t t}+6|\psi|^{2} \psi_{t}\right) \\
& \quad+\gamma\left(\psi_{t t t t}+6|\psi|^{4} \psi+2 \psi^{2} \psi_{t t}^{*}+4 \psi\left|\psi_{t}\right|^{2}\right. \\
& \left.\quad+6 \psi^{*}\left(\psi_{t}\right)^{2}+8|\psi|^{2} \psi_{t t}\right) \\
& \quad-i \delta\left(\psi_{t t t t t}+30|\psi|^{4} \psi_{t}+10 \psi \psi_{t} \psi_{t t}^{*}\right. \\
& \quad+10 \psi_{t}\left|\psi_{t}\right|^{2}+10 \psi \psi_{t}^{*} \psi_{t t}+20 \psi^{*} \psi_{t} \psi_{t t} \\
& \left.\quad+10|\psi|^{2} \psi_{t t t}\right)=0
\end{aligned}
$$

The first two coefficients, $f_{0}$ and $f_{1}$, are generally set to zero, since the corresponding terms simply relate to phase and velocity transformations applied to solutions. Then, Eq. (6) reproduces Eq. (1). To eliminate any possible confusion, we also emphasize here that all the subscripted $A_{j}$ and $B_{j}$ expressions (for $0 \leqslant j \leqslant 5$ ) denote nothing more than the coefficients of the eigenvalue polynomial represented by the $V$ matrix. They have no direct relation to the "hierarchical order" of operators that was mentioned in Sec. I. For instance, the hierarchically fourth-order LPD coefficient $\gamma$ is not limited to $A_{4}$ and $B_{4}$, arising instead in all $V_{j}$ (for $j \leqslant 4$ ) so the zero-curvature equation can adequately reproduce all the LPD terms. This clarification aside, it is evident that setting $\alpha=\gamma=0$ within the Lax pair will reduce generalized Eq. (6) into the specific form of integrable "quintic" equation that we study here, complete with the fundamental NLS operator.

\section{A. The Darboux scheme}

Referring back to the Lax pair formalism in Eq. (2), the elementary version of the Darboux method [36] attempts to find an operator $T$ so $T R$ is compatible with a new $\tilde{U}$ and $\tilde{V}$ Lax pair of interest. In general, these matrices must be of the same structure as the old $U$ and $V$ Lax pair, except with wave function $\psi$ replaced by $\tilde{\psi}$. The idea is that if one possesses a solution $\psi$ to the nonlinear system, along with compatible $R$, then one should also be able to construct a new wave function $\tilde{\psi}$ using the new Lax pair solution $T R$.

This procedure is already well established in the case of the standard NLSE, providing utility for multisoliton [37] and multibreather [38] construction, and nothing appears to change for higher-order equations. Indeed, the simplest "seeding" solution of the quintic NLSE is still $\psi=\psi_{0}=0$, for which vector $R$ can easily be determined. This is particularly true as all $\beta_{j}$ in Eq. (5) become zero, meaning that both $U$ and $V$ are purely diagonal in form. Consequently, Eq. (2) produces what we call "first-order component functions,"

$$
R=\left[\begin{array}{l}
r_{1, j} \\
s_{1, j}
\end{array}\right] .
$$

Here we note that $j$ is an indexation variable, to be used shortly in constructing high-order solutions, and each set of component functions $\left(r_{1, j}, s_{1, j}\right)$ is linked with an individual set of parameters also indexed by $j$, namely eigenvalue $\lambda_{j}$ and axial shifts $\left(x_{j}, t_{j}\right)$.

As the transformation matrix $T$ can be applied multiple times, so, too, can solutions of ever higher order be generated. For the quintic equation, a solution of order $n$ is recursively constructed via

$$
\psi_{n}=\psi_{n-1}+\frac{2\left(\lambda_{n}^{*}-\lambda_{n}\right) s_{n, 1} r_{n, 1}^{*}}{\left|r_{n, 1}\right|^{2}+\left|s_{n, 1}\right|^{2}} .
$$

For $(n, j)=(1,1)$, the substitution of $r_{1,1}$ and $s_{1,1}$ from Eq. (7) into Eq. (8) reveals that $\psi_{0}=0$ is a base on which to construct a soliton solution. For $n>1$, higher-order component functions must first be produced by

$$
\begin{aligned}
r_{n, j}= & J\left[\left(\lambda_{n-1}^{*}-\lambda_{n-1}\right) s_{n-1,1}^{*} r_{n-1,1} s_{n-1, j+1}\right. \\
& +\left(\lambda_{j+n-1}-\lambda_{n-1}\right)\left|r_{n-1,1}\right|^{2} r_{n-1, j+1} \\
& \left.+\left(\lambda_{j+n-1}-\lambda_{n-1}^{*}\right)\left|s_{n-1,1}\right|^{2} r_{n-1, j+1}\right], \\
s_{n, j}= & J\left[\left(\lambda_{n-1}^{*}-\lambda_{n-1}\right) s_{n-1,1} r_{n-1,1}^{*} r_{n-1, j+1}\right. \\
& +\left(\lambda_{j+n-1}-\lambda_{n-1}\right)\left|s_{n-1,1}\right|^{2} s_{n-1, j+1} \\
& \left.+\left(\lambda_{j+n-1}-\lambda_{n-1}^{*}\right)\left|r_{n-1,1}\right|^{2} s_{n-1, j+1}\right],
\end{aligned}
$$

where $J=\left(\left|r_{n-1,1}\right|^{2}+\left|s_{n-1,1}\right|^{2}\right)^{-1}$. In this way, it is possible to nonlinearly superimpose $n$ solitons with $n$ sets of parameters to produce one compound wave function.

\section{EXACT QUINTIC NLSE SOLUTIONS}

\section{A. Single-soliton solution}

As previously stated, all solitonic structures for a governing equation within the NLS hierarchy can be constructed via the Darboux method upon a zero background, as given by $\psi=\psi_{0}=0$. Substituting this into the matrices within Eqs. (4) and (6), remembering that $f_{0}=f_{1}=\alpha=\gamma=0$, the firstorder component functions in Eq. (7) are readily derived from the linear Lax pair system in Eq. (2). These functions of $x$ and $t$ are specifically given as

$$
\begin{aligned}
& r_{1,1}=\exp \left[i x \lambda_{1}^{2}\left(16 \delta \lambda_{1}^{3}+1\right)+i \lambda_{1} t\right], \\
& s_{1,1}=\exp \left[-i x \lambda_{1}^{2}\left(16 \delta \lambda_{1}^{3}+1\right)-i \lambda_{1} t\right],
\end{aligned}
$$

where it is interesting to note the reciprocity with $s_{1,1}=1 / r_{1,1}$. Additionally, an arbitrary phase term can be inserted into the $r$ and $s$ functions, arising as a constant of integration, but it is ignored here for convenience of expression. As mentioned in Sec. II A, the integration constant can also take the form of axial shifts, via the replacement of $x$ and $t$ with $x-x_{1}$ and $t-t_{1}$, respectively. In the related case of breathers, the effect of these axial shifts can be unintuitive and dramatic [21,39], but they are henceforth ignored in this work, as they do not significantly affect the solitons. We also note that eigenvalue $\lambda_{1}$ is allowed to be complex, unless a restriction is explicitly stated.

It is now possible to use the seed $\psi_{0}=0$ and the component functions in Eq. (10) to generate the first nontrivial solution $\psi_{1}$ via the transformation in Eq. (8). The resulting structure happens to be a first-order soliton of Eq. (1), recalling that only $\delta$ is nonzero for our quintic equation. Explicitly, with $\lambda_{1}=a+i b$, the solution is

$$
\psi_{1}(x, t)=2 i b \exp [-2 i \Omega(x, t)] \operatorname{sech}[2 b(t+x \vartheta)],
$$

with the coefficient

$$
\vartheta=2\left[8 \delta\left(5 a^{4}-10 a^{2} b^{2}+b^{4}\right)+a\right]
$$


and the phase factor

$$
\Omega(x, t)=x\left[a^{2}-b^{2}+16 a \delta\left(a^{4}-10 a^{2} b^{2}+5 b^{4}\right)\right]+a t .
$$

The soliton amplitude is simply $2|b|$, i.e., the same as for the NLSE soliton. Its width along the $t$ axis also does not change. However, now the soliton velocity is

$$
v=-\vartheta=-2\left[8 \delta\left(5 a^{4}-10 a^{2} b^{2}+b^{4}\right)+a\right],
$$

which differs from the velocity of the NLSE soliton.

When $\delta=0$, Eq. (11) is a soliton of the fundamental NLSE. In that scenario, its velocity, $-2 a$, is completely determined by the real part, $a$, of eigenvalue $\lambda_{1}$. Thus, a purely imaginary eigenvalue corresponds to straight propagation of the soliton along the $x$ axis. For the quintic equation, the soliton has nonzero velocity even for $a=0$, viz. $v=-16 \delta b^{4}$. Then the phase term $\exp \left(2 i b^{2} x\right)$ evolves along the $x$ axis and does not depend on $t$. Thus, the directions of phase evolution and soliton propagation generally differ. This creates interference patterns for colliding solitons that would be unusual in the NLSE case (e.g., see Fig. 2).

For purely imaginary eigenvalues, terms arising from hierarchical orders that are even (e.g., the NLS and LPD operators) solely affect the phase factor, while terms related to odd hierarchical orders (e.g., the Hirota and quintic operators) affect the group velocity alone. An example of a first-order soliton of the quintic equation is shown in Fig. 1, with $\delta=\frac{1}{64}, a=1$, and $b=1$. The imaginary part of eigenvalue $\lambda_{1}$ determines the height of the structure, given as $\left|\psi_{1}(0,0)\right|=$ $2|b|$, so the displayed wave function has an amplitude of 2 . The soliton velocity in this case is -1 , which corresponds to the structure in Fig. 1 propagating left along the $t$ axis (with $|v|=1$ ) for increasing values of evolution variable $x$. As can be seen, even a very small $\delta$ significantly influences the soliton velocity.

\section{B. General second-order soliton}

As detailed in Sec. II A, it is possible to combine two first-order solitons in nonlinear superposition to create a second-order soliton. Because this involves two "components," the first-order component functions from Eq. (10) must be

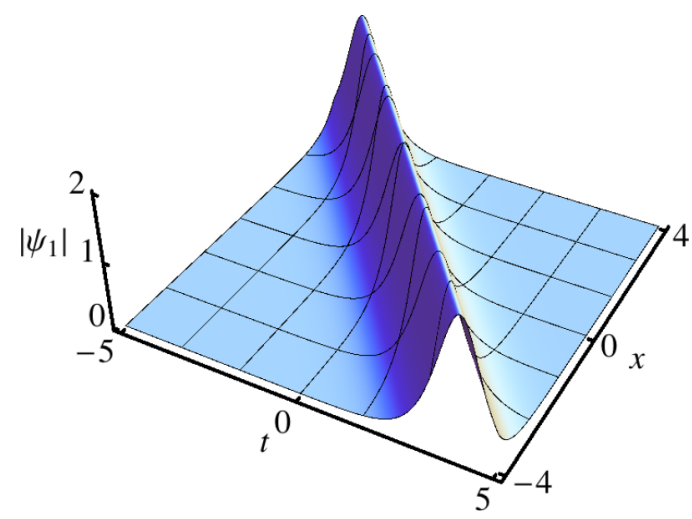

FIG. 1. (Color online) A soliton solution of the quintic equation as given by Eq. (11). Parameters are $\delta=2^{-6}$ and $\lambda=1+i(a=1$ and $b=1$ ). Propagation direction is along the $x$ axis. generalized as

$$
\begin{aligned}
& r_{1, j}=\exp \left[i x \lambda_{j}^{2}\left(16 \delta \lambda_{j}^{3}+1\right)+i \lambda_{j} t\right], \\
& s_{1, j}=\frac{1}{r_{1, j}}
\end{aligned}
$$

where $j=1$ or 2 , depending on the first-order soliton that is being referenced. Mathematically, the superposition procedure involves using Eq. (9) to generate second-order component functions $r_{2,1}$ and $s_{2,1}$ before employing Eq. (8) with $\psi_{1}$ from Eq. (11) to generate the new solution $\psi_{2}$.

The result is

$$
\begin{aligned}
\psi_{2}(x, t)= & 2 e^{-2 i\left(\Omega_{1}+\Omega_{2}\right)} \frac{N_{2}}{D_{2}} \\
N_{2}= & i b_{2}\left[\left(a_{1}-a_{2}\right)^{2}-b_{1}^{2}+b_{2}^{2}\right] e^{2 i \Omega_{1}} \cosh \left(2 \Theta_{1}\right) \\
& +i b_{1}\left[\left(a_{1}-a_{2}\right)^{2}+b_{1}^{2}-b_{2}^{2}\right] e^{2 i \Omega_{2}} \cosh \left(2 \Theta_{2}\right) \\
& -2\left(a_{1}-a_{2}\right) b_{1} b_{2} S_{1} \\
D_{2}= & {\left[\left(a_{1}-a_{2}\right)^{2}+b_{1}^{2}+b_{2}^{2}\right] S_{2}-2 b_{1} b_{2} S_{3} } \\
S_{1}= & e^{2 i \Omega_{1}} \sinh \left(2 \Theta_{1}\right)-e^{2 i \Omega_{2}} \sinh \left(2 \Theta_{2}\right), \\
S_{2}= & \cosh \left(2 \Theta_{1}\right) \cosh \left(2 \Theta_{2}\right), \\
S_{3}= & \cos \left[2\left(\Omega_{1}-\Omega_{2}\right)\right]+\sinh \left(2 \Theta_{1}\right) \sinh \left(2 \Theta_{2}\right),
\end{aligned}
$$

with

$$
\begin{aligned}
\lambda_{j} & =a_{j}+i b_{j}, \\
\Omega_{j} & =a_{j} t+x\left[a_{j}^{2}-b_{j}^{2}+16 a_{j} \delta\left(a_{j}^{4}-10 a_{j}^{2} b_{j}^{2}+5 b_{j}^{4}\right)\right], \\
\Theta_{j} & =b_{j}\left[t+2 x\left[a_{j}+8 \delta\left(5 a_{j}^{4}-10 a_{j}^{2} b_{j}^{2}+b_{j}^{4}\right)\right]\right] .
\end{aligned}
$$

This solution, $\psi_{2}(x, t)$ depends on the fifth-order equation coefficient $\delta$ and thus differs from the known NLSE result. While complicated in appearance, the form of this expression shares similarities with that of the first-order soliton. For instance, the phase factor of each component, as given by Eq. (13), is easily discernible as $\Omega_{j}$. Each factor $\Theta_{j}$ is also essentially the sech argument in Eq. (11), complete with indexed versions of the coefficient $\vartheta$ shown in Eq. (12). Thus Eq. (15) describes two first-order solitons passing through the origin, generally colliding at an angle for arbitrary $\lambda_{j}$. The height of this intersection is the amplitude sum or difference of both individual solitons, depending on constructive or destructive interference, and is written as $\left|\psi_{2}(0,0)\right|=2 \mid b_{1}+$ $b_{2} \mid$. In the case of positive equal unitary values, $b_{1}=b_{2}=1$, the maximum amplitude is 4 . This result is indeed confirmed by Fig. 2, which depicts two solitons with arbitrary velocities colliding. The interference pattern here is more complicated than the case involving the collision of NLSE solitons. This happens because the direction of phase evolution and pulse propagation for each soliton may differ significantly.

\section{Beating second-order soliton}

It is worth examining the second-order quintic soliton in Eq. (15) with greater care. For instance, when $\delta=0$, the expression reduces to a solution of the standard NLSE. For distinct purely imaginary eigenvalues $\lambda_{1}=i b_{1}$ and $\lambda_{2}=i b_{2}$, this "beating" structure consists of two solitons in perfect alignment along evolution axis $x$. This is the well-known 


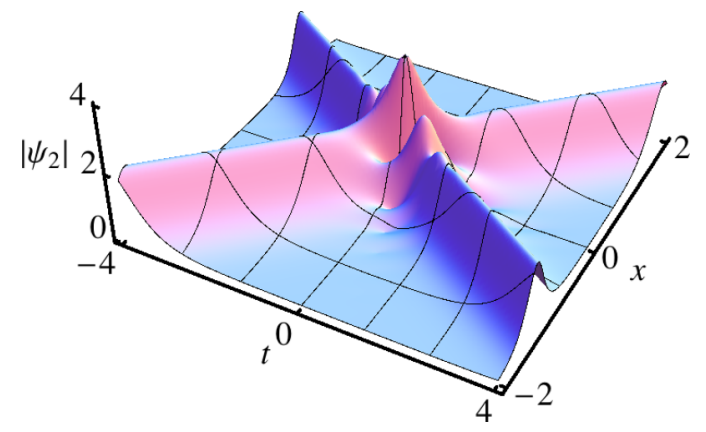

FIG. 2. (Color online) A two-soliton solution of the quintic equation as given by Eq. (15). Parameters are $\delta=0.001, \lambda_{1}=-3.5+i$, and $\lambda_{2}=-1+i$, so as to depict a collision of two solitons. Propagation direction is along the $x$ axis.

"higher-order soliton" of Satsuma and Yajima [40]. As discussed previously, this overlap arises because each soliton velocity becomes solely dependent on the real part, $a_{j}$, of the eigenvalue, as is made explicit in Eq. (12). Thus the two components are only coincident when $a_{1}=a_{2}$.

Soliton alignment is no longer simple when the quintic operator from Eq. (1) comes into play, considering that the axial alignment angle of each individual component is now dependent on its amplitude. While general soliton collisions of finite but significant duration still impart degrees of modulation [29], we restrict the "beating soliton" label to components that are perfectly aligned for all $x$. This is why the inclusion of $a_{1}$ and $a_{2}$ is important, so velocity adjustments can be made to realize coincident first-order solitons. This alignment is only possible if

$5 a_{1}^{4}-10 a_{1}^{2} b_{1}^{2}+b_{1}^{4}+\frac{a_{1}}{8 \delta}=5 a_{2}^{4}-10 a_{2}^{2} b_{2}^{2}+b_{2}^{4}+\frac{a_{2}}{8 \delta}$,

which happens to confirm the aforementioned standard NLSE condition of $a_{1}=a_{2}$ when $\delta=0$. But even when $\delta \neq 0$, it is possible to, first, select the amplitudes of the two-component solitons by fixing $b_{1}$ and $b_{2}$, then choose the velocity of one structure via the selection of $a_{2}$, and, finally, solve Eq. (17) to determine the appropriate $a_{1}$ value that achieves solitonic alignment.

Because of the quartic polynomials involved, the function $a_{1}\left(b_{1}, a_{2}, b_{2}\right)$ is omitted here, due to its complexity. Nonetheless, there are four possible solutions for each set of parameters, two of which are generally real. As $a_{1}$ cannot have an imaginary part by definition, only those two values are acceptable for attaining soliton alignment. The end result is that the second-order soliton in Eq. (15) becomes an angled beating structure, with the choice of $a_{1}$ typically determining whether the beating frequency is high or low. An example of the latter case is depicted in Fig. 3, composed of a $b_{1}=1$ soliton coincident with a $b_{2}=1 / 2$ soliton. Correspondingly, the peaks that denote constructive interference have an amplitude of 3 .

An interesting case now arises when considering the nonlinear superposition of two aligned solitons with equal amplitudes (i.e., $b_{1}=b_{2}$ ). In the standard NLSE case, the alignment condition and this restriction require the eigenvalues to be exactly identical (i.e., $\lambda_{1}=\lambda_{2}$ ). The Darboux process does not allow this and a "degenerate" limit needs to be taken, the result of which will be detailed shortly. In contrast, the

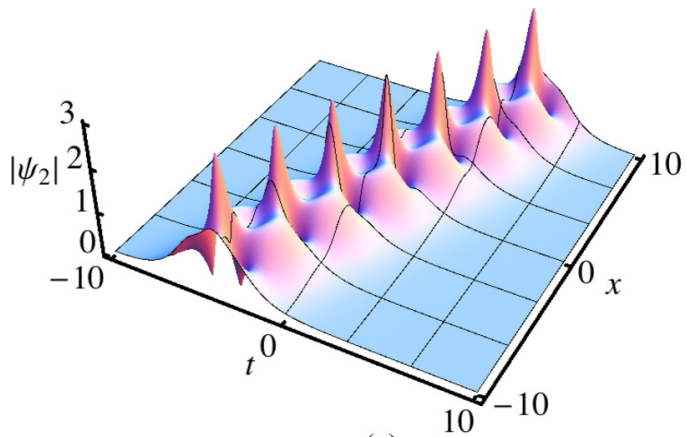

(a)

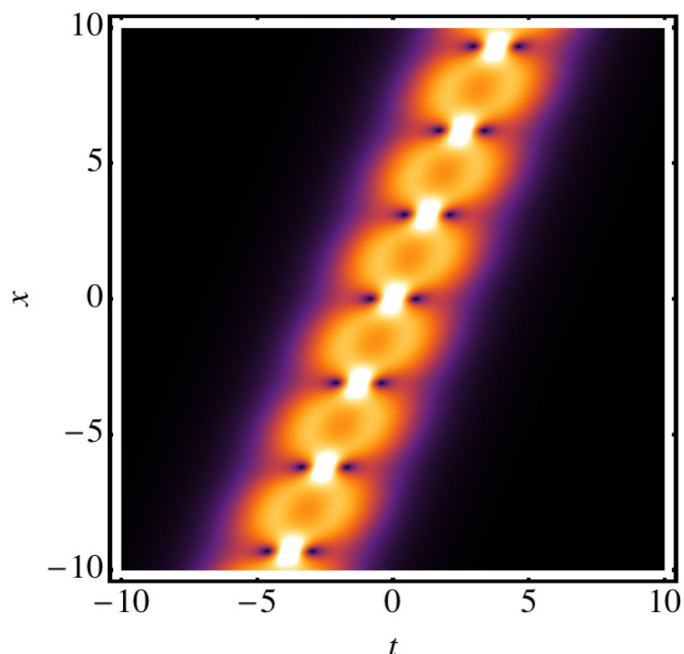

(b)

FIG. 3. (Color online) A second-order periodic soliton of the quintic equation as given by Eq. (15). Parameters are $\delta=2^{-6}, \lambda_{1} \approx$ $-0.251892+i$, and $\lambda_{2}=-1 / 5+i / 2$ to depict beating between two solitons. Propagation direction is along the $x$ axis. (a) Surface diagram. (b) Density diagram.

additional quintic operator allows for two "aligned" scenarios on account of the two real solutions to Eq. (17). One is the upcoming degenerate case with $a_{1}=a_{2}$, but the alternative involves $a_{1} \neq a_{2}$. Indeed, for an arbitrary $\delta$ value, this is generally nothing more than a high beating-frequency secondorder soliton, as shown in Fig. 4. However, we will soon discuss what happens as $\delta$ approaches zero.

\section{Degenerate two-soliton solution}

Degenerate solutions are those that occur when two eigenvalues coincide [20]. However, the Darboux scheme does not give an explicit answer when eigenvalues are strictly equal, a result related to the requirement for asymmetry [41]. To surmount this, a $\lambda_{2} \rightarrow \lambda_{1}$ limit must be applied to the general second-order soliton solution in Eq. (15). The result is

$$
\begin{aligned}
\psi_{2}(x, t) & =-8 i b e^{-2 i \Omega} \frac{N_{2}}{D_{2}}, \\
N_{2} & =P_{1} \sinh (2 \Theta)-\left(1+i P_{2}\right) \cosh (2 \Theta), \\
D_{2} & =1+2\left(P_{1}^{2}+P_{2}^{2}\right)+\cosh (4 \Theta), \\
P_{1} & =2 b\left\{t+2 x\left[a+40 \delta\left(a^{4}-6 a^{2} b^{2}+b^{4}\right)\right]\right\}, \\
P_{2} & =4 b^{2} x\left[1+160 a \delta\left(a^{2}-b^{2}\right)\right],
\end{aligned}
$$




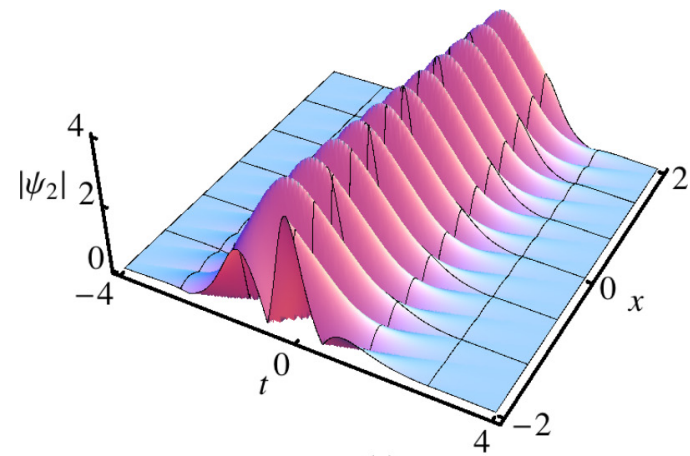

(a)

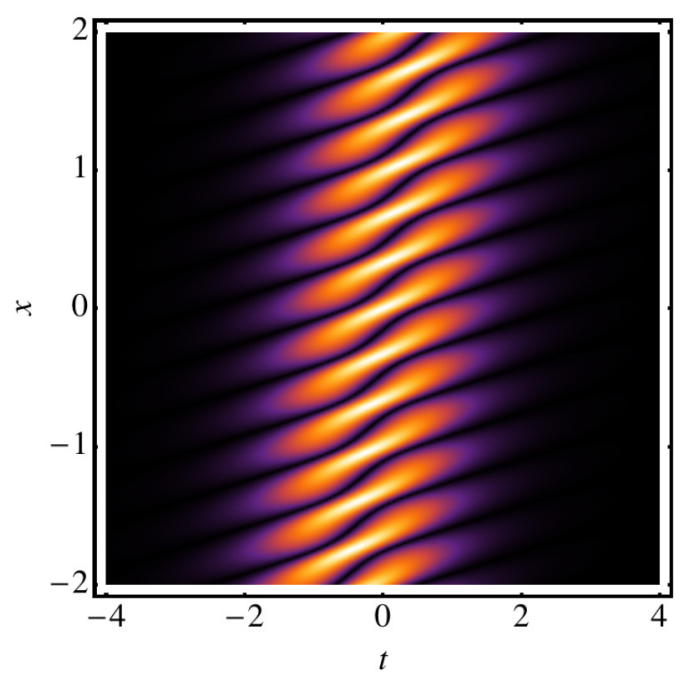

(b)

FIG. 4. (Color online) A second-order periodic soliton of the quintic equation as given by Eq. (15). Parameters are $\delta=2^{-6}$, $\lambda_{1} \approx-1.67702+i$, and $\lambda_{2}=-1 / 5+i$ to depict beating between two equal-amplitude solitons. Propagation direction is along the $x$ axis. (a) Surface diagram. (b) Density diagram.

with

$$
\begin{aligned}
& \lambda=a+i b, \\
& \Omega=a t+x\left[a^{2}-b^{2}+16 a \delta\left(a^{4}-10 a^{2} b^{2}+5 b^{4}\right)\right], \\
& \Theta=b\left\{t+2 x\left[a+8 \delta\left(5 a^{4}-10 a^{2} b^{2}+b^{4}\right)\right]\right\},
\end{aligned}
$$

where the subscripted index has been dropped from all parameters, as the soliton components are identical in eigenvalue.

Given this expression, an example of a degenerate soliton is shown in Fig. 5. Both components in this image possess the same eigenvalue as the single first-order structure in Fig. 1, which means that the two solitons also share the same velocity. It is thus unusual that the solitons seem spatiotemporally parallel instead of coincident, diverging from this behavior only once to intersect at the origin. However, this degenerate structure is actually nothing more than an extreme case of a beating soliton.

In fact, closer inspection of the wave function in Fig. 3 reveals "craters" between every pair of peaks. It follows then that, as $\lambda_{2}$ approaches $\lambda_{1}$ and the beating frequency decreases, the points of constructive interference spread apart and the craters themselves stretch out. In the $\lambda_{2} \rightarrow \lambda_{1}$ limit, only

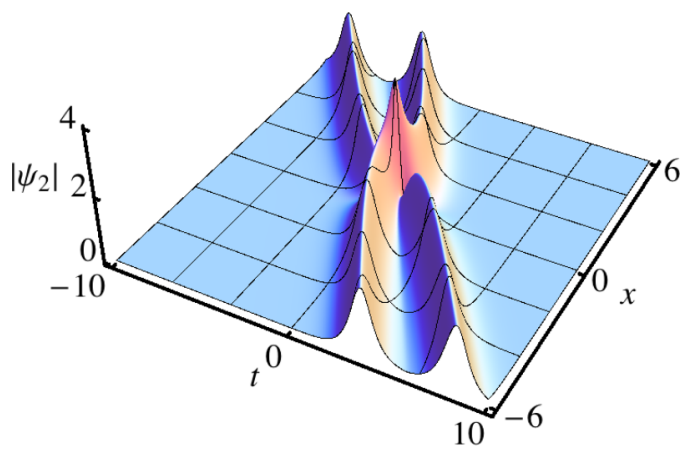

(a)

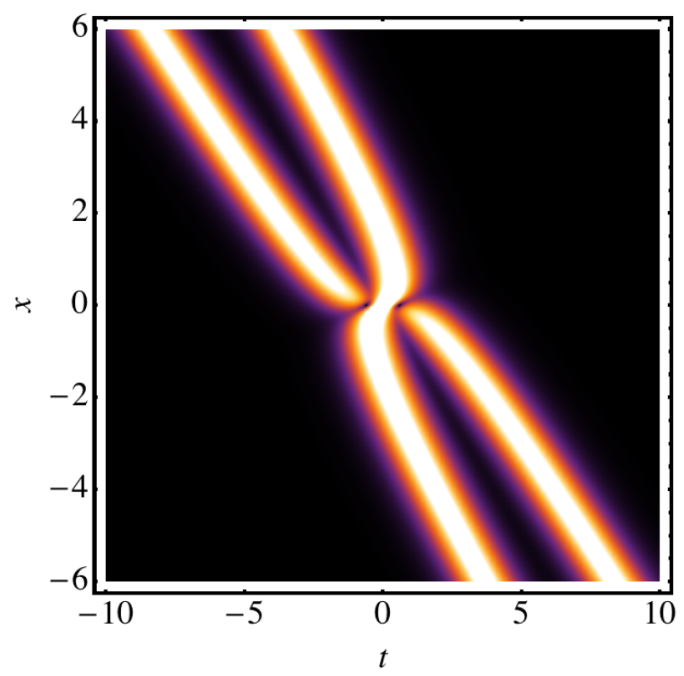

(b)

FIG. 5. (Color online) A two-soliton degenerate solution of the quintic equation as given by Eq. (18). Parameters are $\delta=2^{-6}$ and $\lambda=1+i(a=1$ and $b=1)$. Propagation direction is along the $x$ axis. (a) Surface diagram. (b) Density diagram.

one intersection peak remains in the finite domain, leaving behind crater structures of infinite length that appear as parallel solitons. As a side note, it is also worth mentioning that a deep relationship exists between the peak of a second-order degenerate soliton and a first-order "rogue wave" [42], but we leave that discussion limited here.

This degenerate case can be considered one way that the NLSE hierarchy "cheats" the restriction on intersecting equalamplitude solitons having the same velocity. As mentioned in Sec. III C, the other way is to have the two first-order solitons beat, generally with extremely high frequency. Indeed, as $\delta$ becomes substantially smaller, the periodicity of the resulting interference pattern seems to decrease. It follows that, in a numerically applied $\delta \rightarrow 0$ limit, the envelope of the beating structure appears to approximate a first-order soliton with double amplitude, in a way that is similar to that of a $|\cos (\omega x)|$ envelope approaching the constant 1 for large $\omega$ values. In effect, the two solitons are attempting to linearly superimpose within the restrictions of nonlinear superposition, and this situation is shown in Fig. 6. The depicted oscillations in Figs. 6(a) and 6(b) are cross-sectional profiles along $x$ and $t$, respectively, of such a wave function, the surface of which would be a higher-frequency version of Fig. 4. This structure 


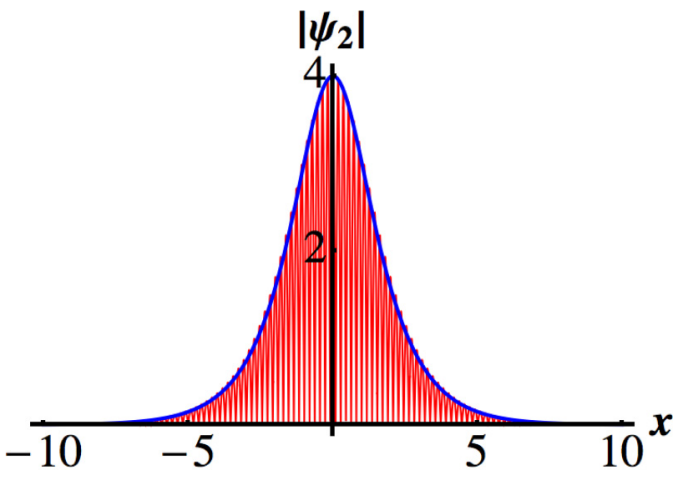

(a)

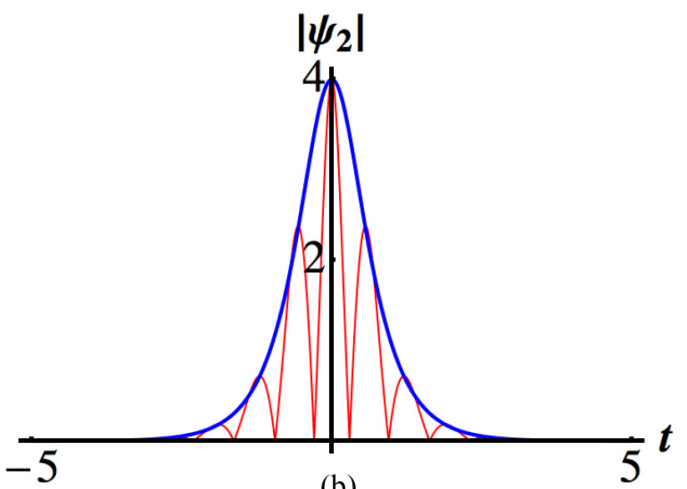

(b)

FIG. 6. (Color online) Cross-sectional cuts of both a doubleamplitude first-order soliton (the smooth blue lines) and a secondorder equal-amplitude beating soliton (the oscillating red lines). The quintic parameter is $\delta=2^{-12}$. Eigenvalue of double-amplitude soliton is $\lambda=-1 / 5+i$. Eigenvalues of beating structure are $\lambda_{1} \approx-4.75617+i$ and $\lambda_{2}=-1 / 5+i$. (a) Cross sections along $x$. (b) Cross sections along $t$.

is significant as it appears to have no analog in the standard NLSE case. Presumably, when $\delta=0$, the beating frequency becomes infinite and the solution can no longer exist.

However, we do note here that the addition of the quintic operator alone may not be necessary for the existence of this new solution. Any terms that modify velocity in such a manner that Eq. (17) is left nontrivial should allow for the construction of this special case. This means that the combination of the standard NLSE and the Hirota operator should also allow for a beating structure composed of coincident equal-amplitude solitons.

\section{E. Third-order solitons}

We do not present the explicit expression for a third-order soliton here due to its written length. Nonetheless, it is standard fare to generate these solutions via the recursive formulas of the Darboux scheme, as detailed in Eqs. (8) and (9). Consequently, a typical three-soliton collision is displayed in Fig. 7. As shifts are still ignored in this work, all components constructively interfere at the origin and attain a maximum amplitude of $\psi_{3}(0,0)=2\left(b_{1}+b_{2}+b_{3}\right)$, provided that $b_{j} \geqslant 0$ for all $j$. Given the component eigenvalues of the depicted wave function, the intersection peak of the structure reaches a height of 4.5 .

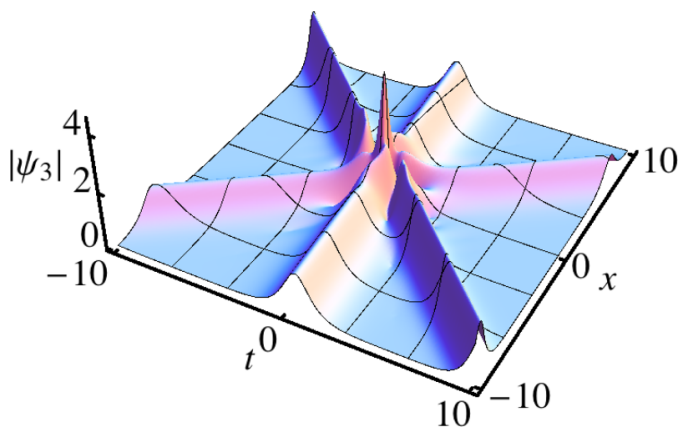

(a)

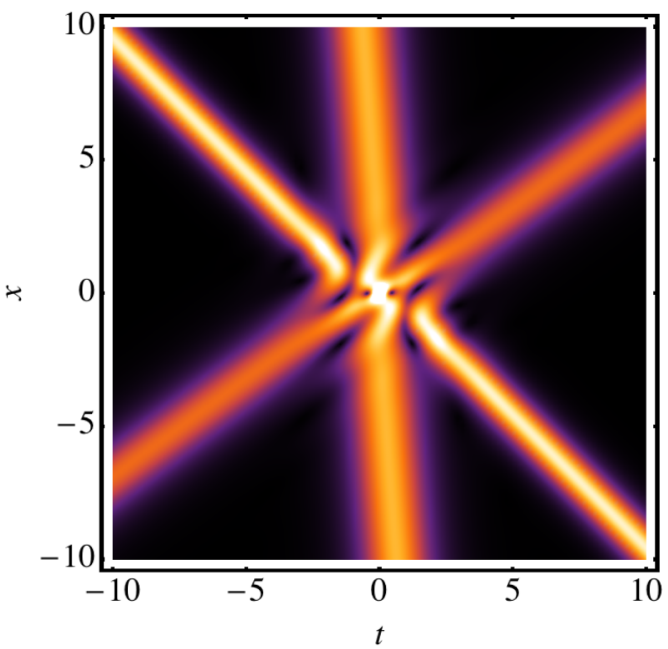

(b)

FIG. 7. (Color online) Three-soliton solution, with parameters $\delta=2^{-6}, \lambda_{1}=-1+i / 2, \lambda_{2}=3 i / 4$, and $\lambda_{3}=1+i$. Propagation direction is along the $x$ axis. (a) Surface diagram. (b) Density diagram.

It is of course possible to align three solitons by use of the same strategy in Sec. III C. An example of a third-order beating structure is shown in Fig. 8, where $a_{1}$ and $a_{3}$ were determined following the fixing of $a_{2}$ and $b_{2}$. The wave function thus has the same velocity as the second-order solution in Fig. 3 but now features an additional component. With a value of $b_{3}=3 / 2$, this third soliton adds to the central peak via constructive interference to reach a maximum amplitude of 6 .

As previously noted, the points of intersection for an order $n$ soliton are actually order $n-1$ structures from a so-called breather family of solutions [42]. Indeed, a chain of first- and second-order rogue waves are clearly visible in Fig. 8(b). It should also be noted that, while the appearance of a firstorder breather in the intersection of two beating solitons is periodic by definition, as shown in Fig. 3, the regularity of this second-order breather chain in Fig. 8 appears to be gradually breaking down away from the origin. Indeed, a more arbitrary selection of $b_{j}$ values makes this explicit in Fig. 9. Points of intersection adjacent to and near the central fused peak take the form of nonuniform second-order rogue wave triplets [43], as best visualized in Fig. 9(a). The implication here is that there is a low likelihood of any new intersection down the line ever fusing perfectly again. 


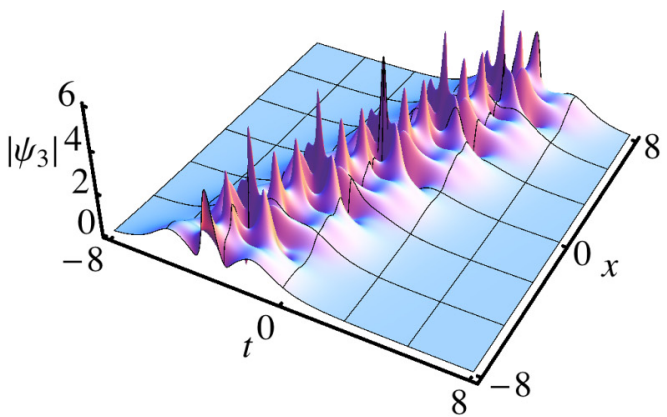

(a)

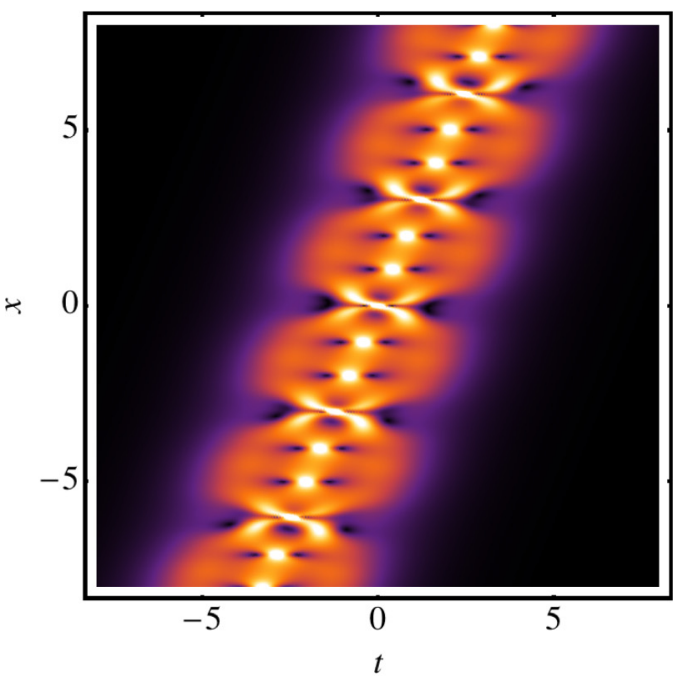

(b)

FIG. 8. (Color online) Third-order beating soliton, with parameters $\delta=2^{-6}, \lambda_{1} \approx-0.251892+i, \lambda_{2}=-1 / 5+i / 2$, and $\lambda_{3} \approx$ $-0.400809+3 i / 2$. Propagation direction is along the $x$ axis. (a) Surface diagram. (b) Density diagram.

It follows that structural periodicity is difficult to attain for a third-order soliton. In the case of second-order breathers, modulation frequencies need to be in integer ratio for this to occur [41]. Similarly, to achieve this type of regularity with three coincident solitons, it is likely that the beating frequency between components 1 and 2 needs to be in integer ratio with the beating frequency between components 2 and 3 . To complicate matters, the quintic operator generally twists solitonic interference patterns with respect to the path along which they occur, as is most apparent with the angled striations in Fig. 4(b). This means that the spatiotemporal direction of solitonic phase oscillation in Eq. (13) needs to be projected onto the spatiotemporal direction in which the soliton travels, with its velocity given by Eq. (12). Simply speaking, the phase exponent and the group velocity of the solitons for higher-order equations are not the same.

As this is somewhat involved, we do not pursue the issue further here. It is worth noting, though, that the components of the structure in Fig. 9 possess similar eigenvalues. Accordingly, the beating frequencies are small and the solitons appear to intersect less often than in the case of Fig. 8. Moreover, from a localized point of view, the shape of a degenerate third-order soliton is evident. Three components

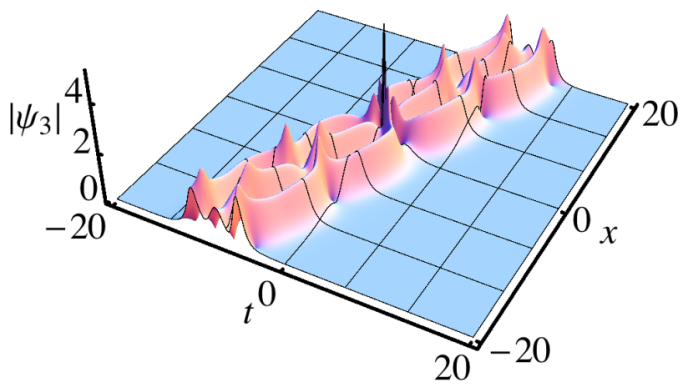

(a)

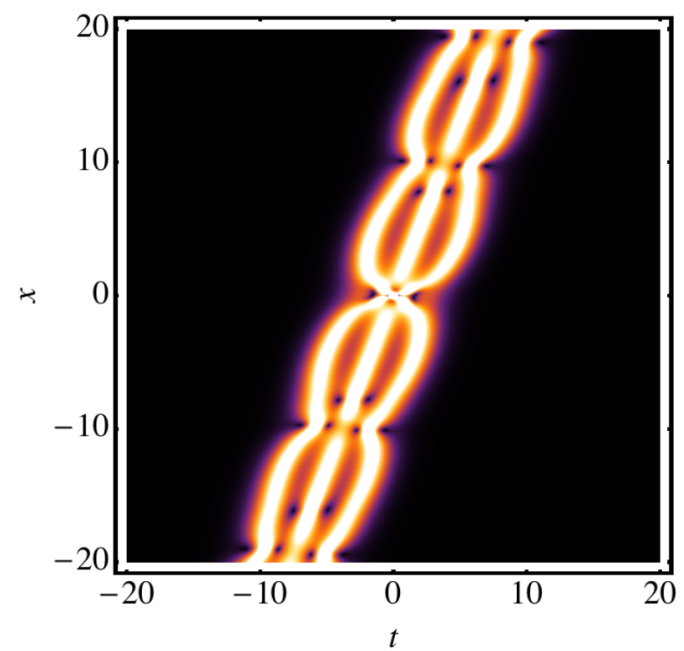

(b)

FIG. 9. (Color online) Third-order beating soliton, with parameters $\delta=2^{-6}, \lambda_{1} \approx-0.244981+i, \lambda_{2}=-1 / 5+i / \sqrt{2}$, and $\lambda_{3} \approx$ $-0.219388+i \sqrt{3} / 2$. Propagation direction is along the $x$ axis. (a) Surface diagram. (b) Density diagram.

with parallel appearance are expected, colliding only once, to give a "pitchfork" arrangement on either side of the origin.

\section{CONCLUSION}

We have studied the fifth-order nonlinear equation in the NLS hierarchy. Its integrability is a corollary of its ability to be expressed in Lax pair form, while the system itself arises naturally when the zero-curvature equation is applied to both a $2 \times 2$ AKNS matrix and a corresponding matrix that is quintic in eigenvalue. Accordingly, we have provided explicit expressions for the Lax pair of this equation.

By using the Darboux scheme, we have then derived the basic one-soliton solution and have nonlinearly superimposed two such components into a general eigenvaluedependent structure. Appropriate choice of parameters allows for the alignment of individual solitons, despite amplitudedependence arising with velocities due to the additional quintic NLS operator. Consequently, we have examined so-called second-order beating structures and their extreme cases. In the limit of zero beating frequency, the second-order degenerate soliton forms with only one intersection at the origin, despite both components sharing the same velocity. Notably, the quintic operator also allows for a high beating-frequency wave function composed of equal-amplitude solitons, which does not appear to have an analog in the standard NLSE. 
Furthermore, this investigation has involved higher-order soliton structures that are conveniently constructed with the Darboux procedure. It is evident that the peaks formed from solitonic intersection in these cases resemble a related breather family of solutions. Subsequently, it is indicated that an integer ratio between beating frequencies is key to ensuring periodic regularity of the resulting structures.

\section{ACKNOWLEDGMENTS}

The authors acknowledge the support of the Australian Research Council (Discovery Project No. DP140100265). N.A. and A.A. acknowledge support from the Volkswagen Stiftung and A.C. acknowledges Endeavour Postgraduate Award support.
[1] A. R. Osborne, Nonlinear Ocean Waves and the Inverse Scattering Transform (Elsevier, Amsterdam, 2010).

[2] N. J. Zabusky and M. D. Kruskal, Phys. Rev. Lett. 15, 240 (1965).

[3] A. S. Davydov, J. Theor. Biol. 66, 379 (1977).

[4] A. Porter and N. F. Smyth, J. Fluid Mech. 454, 1 (2002).

[5] D. R. Scott and D. J. Stevenson, Geophys. Res. Lett. 11, 1161 (1984).

[6] A. Maccari, Electron. J. Theor. Phys. 3, 39 (2006).

[7] A. Hasegawa and F. Tappert, Appl. Phys. Lett. 23, 142 (1973).

[8] L. F. Mollenauer, R. H. Stolen, and J. P. Gordon, Phys. Rev. Lett. 45, 1095 (1980).

[9] M. J. Potasek, Phys. Lett. A 154, 449 (1991).

[10] S. B. Cavalcanti, J. C. Cressoni, H. R. da Cruz, and A. S. Gouveia-Neto, Phys. Rev. A 43, 6162 (1991).

[11] M. Trippenbach and Y. B. Band, Phys. Rev. A 57, 4791 (1998).

[12] G. P. Agrawal, Nonlinear Fiber Optics (Optics and Photonics), 5th ed. (Academic Press, San Diego, 2012).

[13] J. M. Dudley and J. R. Taylor (eds.), Supercontinuum Generation in Optical Fibers (Cambridge University Press, Cambridge, 2010).

[14] N. Akhmediev and M. Karlsson, Phys. Rev. A 51, 2602 (1995).

[15] D. Anderson and M. Lisak, Phys. Rev. A 27, 1393 (1983).

[16] S. Roy, S. K. Bhadra, and G. P. Agrawal, Opt. Commun. 282, 3798 (2009).

[17] M. N. Zambo Abou'ou, P. Tchofo Dinda, C. M. Ngabireng, S. Pitois, and B. Kibler, Phys. Rev. A 87, 033803 (2013).

[18] A. Ankiewicz, Y. Wang, S. Wabnitz, and N. Akhmediev, Phys. Rev. E 89, 012907 (2014).

[19] I. P. Christov, Phys. Rev. A 60, 3244 (1999).

[20] N. Akhmediev and A. Ankiewicz, Solitons: Nonlinear Pulses and Beams, Vol. 5 of Optical and Quantum Electronics (Chapman \& Hall, London, 1997), Chaps. 3 and 4.

[21] D. J. Kedziora, A. Ankiewicz, and N. Akhmediev, Phys. Rev. E 88, 013207 (2013).

[22] R. Hirota, J. Math. Phys. 14, 805 (1973).

[23] A. Ankiewicz, J. M. Soto-Crespo, and N. Akhmediev, Phys. Rev. E 81, 046602 (2010).
[24] R. Sahadevan and L. Nalinidevi, J. Nonlin. Math. Phys. 17, 379 (2010).

[25] M. Lakshmanan, K. Porsezian, and M. Daniel, Phys. Lett. A 133, 483 (1988).

[26] K. Porsezian, M. Daniel, and M. Lakshmanan, J. Math. Phys. 33, 1807 (1992).

[27] K. Porsezian, Phys. Rev. E 55, 3785 (1997).

[28] T. Kano, J. Phys. Soc. Jpn. 58, 4322 (1989).

[29] A. Ankiewicz and N. Akhmediev, Phys. Lett. A 378, 358 (2014).

[30] S. M. Hoseini and T. R. Marchant, Wave Motion 44, 92 (2006).

[31] Pan Wang, Bo Tian, Wen-Jun Liu, Qi-Xing Qu, Min Li, and Kun Sun, Eur. Phys. J. D 61, 701 (2011).

[32] R. Sahadevan and L. Nalinidevi, J. Math. Phys. 50, 053505 (2009).

[33] V. E. Zakharov and A. B. Shabat, J. Exp. Theor. Phys. 34, 62 (1972).

[34] A. Degasperis, A. P. Fordy, and M. Lakshmanan (eds.), Nonlinear Evolution Equations: Integrability and Spectral Methods (Manchester University Press, Manchester, 1990).

[35] M. J. Ablowitz, D. J. Kaup, A. C. Newell, and H. Segur, Stud. Appl. Math. 53, 249 (1974).

[36] V. B. Matveev and M. A. Salle, Darboux Transformations and Solitons (Springer-Verlag, Berlin, 1991).

[37] N. Akhmediev and N. V. Mitskevich, IEEE J. Quant. Electron. 27, 849 (1991)

[38] N. Akhmediev, V. I. Korneev, and N. V. Mitskevich, Zh. Eksp. Teor. Fiz. (USSR) 94, 159 (1988) [Sov. Phys. JETP 67, 89 (1988)].

[39] D. J. Kedziora, A. Ankiewicz, and N. Akhmediev, Phys. Rev. E 84, 056611 (2011).

[40] J. Satsuma and N. Yajima, Suppl. Progr. Theor. Phys. 55, 284 (1974).

[41] D. J. Kedziora, A. Ankiewicz, and N. Akhmediev, Phys. Rev. E 85, 066601 (2012).

[42] D. J. Kedziora, A. Ankiewicz, and N. Akhmediev, Eur. Phys. J. Spec. Top. 223, 43 (2014).

[43] A. Ankiewicz, D. J. Kedziora, and N. Akhmediev, Phys. Lett. A 375, 2782 (2011). 